\title{
Nano-wires associated with compositional inhomogeneities in multilayered films
}

\author{
I A Ovid'ko and A G Sheinerman \\ Institute for Problems of Mechanical Engineering, Russian Academy of Sciences, Bolshoj 61, \\ Vasiljevskiy Ostrov, St Petersburg 199178, Russia \\ E-mail: ovidko@def.ipme.ru
}

Received 8 May 2001, in final form 6 August 2001

Published 5 October 2001

Online at stacks.iop.org/JPhysCM/13/9645

\begin{abstract}
A theoretical model is suggested which describes the formation of nano-wires associated with compositional inhomogeneities in multilayered films. General formulae are found relating characteristic length scales of compositional inhomogeneities in film layers and geometric parameters (layer thickness values, misfit parameters) of the multilayered film. The exact relationship between such length scales and parameters is revealed and analysed in detail in the exemplary case of three-layer films.
\end{abstract}

\section{Introduction}

Nanostructured films and coatings exhibit outstanding physical properties widely exploited in contemporary high technologies (see [1-6]). In particular, semiconductor nano-islands (quantum dots) and nano-wires are the subject of intensive theoretical and experimental studies owing to their great technological potential for device applications (see [7-30]). In addition to technologically motivated attention to nano-islands and nano-wires, their examinations are highly interesting for understanding the fundamental nature of nano-scale effects in condensed matter.

One of the effective and promising methods for fabrication of nano-wires exploits the effect of the occurrence of compositional inhomogeneities in polyatomic films (see review [8]). In doing so, a tentatively periodic modulation of the chemical composition in a polyatomic film results from the thermodynamically driven compositional decay of a solid solution, with characteristic scales of compositional inhomogeneities being strongly influenced by misfit stresses. The modulation gives rise to the occurrence of nano-wires which represent tentatively wire-shaped regions with the composition being different from that of the surrounding material and wire diameters being of the order of a few nm [8]. In the situation discussed, the formation of nano-wires is controlled by the following three factors: 
(i) Separation of a pre-existent compositionally homogeneous solid solution into two phases with various chemical compositions. It is driven by a decrease of the thermodynamic potential that characterizes the solid solution.

(ii) Elastic strains occurring in response to the separation-induced spatial variations of the crystal lattice parameter in the film.

(iii) Misfit stresses (generated at the film/substrate interface) which, as with compositionally homogeneous films (see [31-44]), strongly contribute to the total energy density of the heteroepitaxial system and, therefore, influence the compositional inhomogeneities in the film.

These three key factors can be regulated by choosing geometric parameters (e.g., film thickness) and materials of the film and the substrate. As a corollary, it is potentially possible to technologically control and design the characteristics of nano-wires in compositionally inhomogeneous films, with the effects of the three key factors (phase separation, stresses associated with compositional inhomogeneities in film and misfit stresses) on the characteristics of nano-wires being understood and taken into consideration. In recent years, research efforts in this area have focused on nano-wires in one-layer films with compositional inhomogeneities (see [8,45-48]). At the same time, compositional nano-wires can also be fabricated in multilayered composite films (see $[14,49,50]$ ), in which case the characteristics of nano-wires are expected to depend crucially on layer thickness values and misfit parameters as well as on the compositions of the layers. In particular, computer simulations [14] of the structure of multilayered semiconductor films GaAs/InAs and $\mathrm{GaP} / \mathrm{InP}$ with composition modulation have indicated the possibility of regulating the optical properties of these semiconductor films by tuning the frequency and amplitude of the modulation. In the situation discussed, the set of technologically-controlled parameters of multilayered films is richer than that of the one-layer films. This leads to a more effective way to fabricate compositional nano-wires with desired structural and behavioural characteristics in multilayered films, compared to one-layer films. The main aim of this paper is to elaborate a theoretical model which describes the influence of geometric parameters (layer thickness values, misfit parameters) of multilayered films on the formation of compositional nano-wires in such films with particular emphasis on the exemplary case of three-layer films.

\section{Multilayered film with compositional inhomogeneities}

Let us consider a composite system consisting of a semi-infinite substrate (phase $\gamma$ ) and a multilayered film composed of $2 N$ alternate layers $\delta$ and $\gamma$ (figure 1). The film and the substrate are assumed to be isotropic solids with the same value of the Young modulus $E$ and the same value of the Poisson ratio $v$. The $k$ th layer $(\delta$ or $\gamma)$ is characterized by the thickness $t_{k}(k=$ $1, \ldots, 2 N$ ). In the framework of our model, the chemical composition of the phase $\gamma$ (substrate and layers numerated by even integers) is spatially homogeneous. The chemical composition of the layers $\delta$ is assumed to be spatially inhomogeneous, causing a cos-like modulation of the crystal lattice parameter along the $x$-axis parallel to the film/substrate boundary plane $(x, y)$. That is,

$$
\begin{aligned}
& a(x, y, z)=a_{0}\left(1-\varepsilon_{0} \cos \alpha x\right) \quad \text { for layers } \delta \\
& a(x, y, z)=a_{0} \quad \text { for substrate and layers } \gamma
\end{aligned}
$$

where $z$ denotes the coordinate along a normal to the film/substrate boundary plane $(x, y)$, $\varepsilon_{0}$ and $2 \pi / \alpha$ are the amplitude and period of the periodic modulation, respectively. 


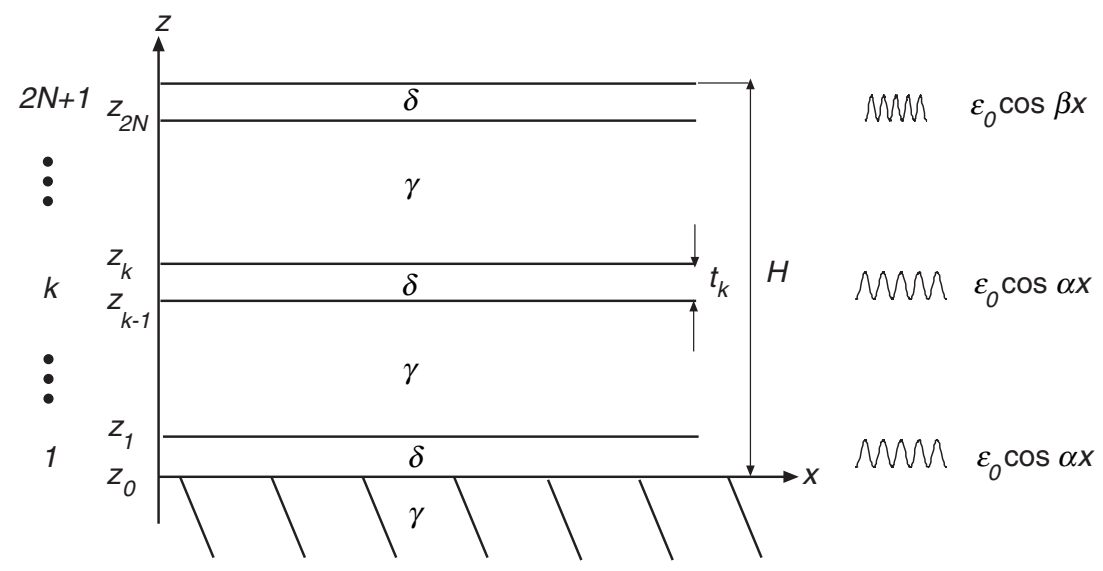

Figure 1. Multilayered film on substrate.

Let us consider the situation where a new layer $\delta$ of thickness $t_{2 N+1}$ is deposited onto the $2 N$ th upper layer $\gamma$ of the multilayered film (figure 1). In doing so, we assume that the crystal lattice parameter $a_{u}$ of the upper layer $\delta$ is modulated as follows:

$$
a_{u}(x, y, z)=a_{0}\left(1-\varepsilon_{0} \cos \beta x\right) .
$$

The frequency $\beta$ of its modulation can, in general, be different from the frequency $\alpha$ that characterizes periodic modulations of the pre-existent layers $\delta$. In the next section we will find the frequency $\beta$ which corresponds to a minimum of the Helmholz free energy of the composite system. The free energy density (per unit volume) of a semiconductor film has the following three constituents: the elastic energy density that characterizes elastic strains, the chemical energy density that characterizes the formation of a compositionally homogeneous solid solution, and the gradient energy density that characterizes compositional (chemical) inhomogeneities [51]. The chemical energy density does not depend on the frequency of the composition modulation. The gradient energy density is negligibly small compared to the elastic energy density [52]. Therefore, the equilibrium spatial frequency of compositional inhomogeneities in a semiconductor film follows from the condition that the elastic energy density of the film is minimal. In addition, in the analysis of compositional inhomogeneities in films, one should take into account the fact that compositional modulation in a growing film occurs via diffusion processes which are commonly intensive only in a thin layer (of thickness of the order of a few interatomic distances) next to the film free surface [52]. In the situation discussed in the next sections of this paper focusing on examinations of compositional inhomogeneities in multilayered films, we assume that the formation of a new layer $\delta$ (that is, its deposition onto the pre-existent multilayered film) does not influence the compositional inhomogeneities within the pre-existent layers $\delta$.

It is interesting to note that periodically modulated compositional inhomogeneities as sources of stresses in films can be treated as some analogue of periodically arranged misfit dislocations. Actually, both misfit dislocations and compositional inhomogeneities create spatially inhomogeneous stress fields that compensate, in part, for misfit stresses in films. In these circumstances, the generation of misfit dislocations and the formation of compositional inhomogeneities play the role of (competing) mechanisms for relaxation of misfit stresses in strained films. In general, misfit dislocations and compositional inhomogeneities can co-exist, in which case the compositional inhomogeneities are capable of causing the generation of such 
non-ordinary misfit dislocation configurations as misfit dislocation dipoles (see experimental data [53] and a model [54]).

\section{Elastic energy of a multilayered film with compositional inhomogeneities}

In order to calculate the elastic energy of the composite system and find the frequency $\beta$ corresponding to its minimum, let us represent the strains $\varepsilon_{i j}$ in the system in question as the sums of the proper strains $\varepsilon_{i j}^{e}$ (that provide coherency at the film/substrate boundary and tend to keep the lattice parameter spatially homogeneous) and strains $\varepsilon_{i j}^{r}$ occurring in the film and the substrate as a result of their relaxation. The stresses $\sigma_{i j}$ in the composite system (figure 1) can be represented as the sums $\sigma_{i j}^{e}+\sigma_{i j}^{r}$ of the corresponding proper stresses $\sigma_{i j}^{e}$ and stresses $\sigma_{i j}^{r}$ resulting from relaxation of the film and the substrate. The strains $\varepsilon_{i j}^{e}$ and stresses $\sigma_{i j}^{e}$ can be written as follows:

$$
\begin{aligned}
& \varepsilon_{i j}^{e}(x, y, z)=\delta_{i j} \varepsilon_{0} \sum_{l=0}^{N}\left[\Theta\left(z-z_{2 l}\right)-\Theta\left(z-z_{2 l+1}\right)\right] \cos p_{l} x \\
& \sigma_{i j}^{e}(x, y, z)=\frac{E}{1-2 v} \varepsilon_{i j}^{e}(x, y, z)
\end{aligned}
$$

where $z_{0}=0, z_{k}=\sum_{m=1}^{k} t_{m}, k=1, \ldots, 2 N+1, p_{l}=\alpha\left(1-\delta_{l, N}\right)+\beta \delta_{l, N}$ and $\Theta(h)$ is the Heaviside function $(\Theta(h)=1$, for $h \geqslant 0 ; \Theta(h)=0$, for $h<0)$.

The strains $\varepsilon_{i j}^{e}$ that exist in the film are represented as the superpositions of the strains $\epsilon_{i j}^{(2 m+1)}=\delta_{i j} \varepsilon_{0} \cos p_{m} x \Theta\left(z-z_{2 m}\right)$, that exist in the film regions consisting of the layers enumerated by $2 m+1,2 m+2, \ldots, 2 N+1(m=0, \ldots, N)$, and the strains $\epsilon_{i j}^{(2 n+2)}=-\delta_{i j} \varepsilon_{0} \cos p_{n} x \Theta\left(z-z_{2 n+1}\right)$, having opposite sign, that exist in the film regions consisting of the layers enumerated by $2 n+2,2 n+3, \ldots, 2 N+1(n=0, \ldots, N-1)$. As a corollary, the strains $\varepsilon_{i j}^{r}$ can be represented as the superpositions of the strains resulting from relaxation of the film/substrate composite system in response to the action of the strains, $\epsilon_{i j}^{(2 m+1)}$ and $\epsilon_{i j}^{(2 n+2)}$, and the consequent coherent matching of the layers $(2 m$ and $2 m+1)$ and $(2 n+1$ and $2 n+2)$, respectively.

Thus, we find the following formulae for the strains $\varepsilon_{i j}^{r}$ :

$$
\varepsilon_{i j}^{r}(x, y, z)=\sum_{l=0}^{2 N}(-1)^{l} \tilde{\varepsilon}_{i j}^{r}\left(x, y, z-z_{l}, H-z_{l}, p_{l}\right)
$$

where $H$ is the film thickness, and $\tilde{\varepsilon}_{i j}^{r}(x, y, z, t, \alpha)$ are the strains resulting from relaxation of a one-layer film with thickness $t$ and a lattice parameter having cos-like modulation along the $x$-axis. The formulae for the strains $\tilde{\varepsilon}_{i j}^{r}$ can be written (from the corresponding formulae [30] for displacement fields used as input) as follows:

$$
\begin{aligned}
\tilde{\varepsilon}_{x x}^{r}(x, y, z, t, \alpha) & =\frac{\alpha(1+v)}{E} \exp (\alpha z) \cos \alpha x\left\{\alpha A_{1}-D+2(1-v) A_{3}+\alpha A_{3} z\right. \\
+ & \left.\frac{D}{2}[\exp (-2 \alpha z)-2 \exp (-\alpha z)+1] \Theta(z)\right\} \\
\tilde{\varepsilon}_{z z}^{r}(x, y, z, t, \alpha) & =\frac{\alpha(1+v)}{E} \exp (\alpha z) \cos \alpha x\left\{\alpha A_{1}-D+2 v A_{3}+\alpha A_{3} z\right. \\
+ & \left.\frac{D}{2}[\exp (-2 \alpha z)+1] \Theta(z)\right\}
\end{aligned}
$$




$$
\begin{gathered}
\tilde{\varepsilon}_{x z}^{r}(x, y, z, t, \alpha)=\frac{\alpha(1+v)}{E} \exp (\alpha z) \cos \alpha x\left\{\alpha A_{1}-D+A_{3}+\alpha A_{3} z\right. \\
\left.-\frac{D}{2}[\exp (-2 \alpha z)-1] \Theta(z)\right\} \\
\tilde{\varepsilon}_{x y}^{r}=\tilde{\varepsilon}_{y y}^{r}=\tilde{\varepsilon}_{y z}^{r} \equiv 0
\end{gathered}
$$

where

$$
\begin{aligned}
& A_{1}=\frac{D}{2 \alpha}[-(1+2 \alpha t) \exp (-2 \alpha t)+2(1+\alpha t) \exp (-\alpha t)+1] \\
& A_{3}=D \exp (-\alpha t)[\exp (-\alpha t)-1] \\
& D=\frac{E \varepsilon_{0}}{\alpha(1-v)}
\end{aligned}
$$

The total elastic energy of the composite system is given by the following formula [30]:

$$
W=\frac{1}{2} \int_{V_{1}} \sigma_{i j}^{e}\left(\varepsilon_{i j}^{e}+\varepsilon_{i j}^{r}\right)
$$

with integration over the film volume $V_{1}$. As a corollary, the elastic energy density (averaged over the film volume) can be written as follows:

$$
\langle w\rangle=\frac{1}{2 H} \int_{0}^{H}\left\langle\sigma_{i j}^{e}\left(\varepsilon_{i j}^{e}+\varepsilon_{i j}^{r}\right)\right\rangle_{x} \mathrm{~d} z
$$

where $\langle\cdots\rangle_{x}$ denotes the averaging over the coordinate $x$. With formulae (4)-(13) substituted into formula (15), we have the elastic energy density $\langle w\rangle$ as a function of parameter $\beta$, the frequency of periodic modulations of the lattice parameter in the new layer $\delta$ deposited onto the multilayered film.

\section{Nano-wires associated with compositional inhomogeneities in three-layer films}

Let us consider the exemplary case of a three-layer film consisting of layers 1 (phase $\delta$ ), 2 (phase $\gamma$ ) and 3 (phase $\delta$ ), that is, the case with deposition of the new layer $\delta$ onto the two-layer film, characterized by $N=1$. The mean elastic energy density $\langle w\rangle$ of the three-layer film/substrate composite can be written as follows:

$$
\langle w\rangle=\left\langle w_{1}\right\rangle+\left\langle w_{3}\right\rangle+\left\langle w_{1-3}\right\rangle
$$

where $\left\langle w_{1}\right\rangle$ and $\left\langle w_{3}\right\rangle$ are the mean energy densities of the stresses associated with the compositional inhomogeneities in the layers 1 and 3 , respectively, and $\left\langle w_{1-3}\right\rangle$ is the mean energy density that characterizes the interaction between the stresses associated with the compositional inhomogeneities in the layers 1 and 3. From formulae (6) and (15) we find the expressions for $\left\langle w_{1}\right\rangle,\left\langle w_{3}\right\rangle$ and $\left\langle w_{1-3}\right\rangle$ to be given as

$$
\begin{aligned}
& \left\langle w_{1}\right\rangle=\frac{1}{2 H} \int_{0}^{H}\left\langle\sigma_{i j}^{(1)}\left[\varepsilon_{i j}^{(1)}+\varepsilon_{i j}^{r}(x, y, z, H, \alpha)-\varepsilon_{i j}^{r}\left(x, y, z-t_{1}, H-t_{1}, \alpha\right)\right]\right\rangle_{x} \mathrm{~d} z \\
& \left\langle w_{3}\right\rangle=\frac{1}{2 H} \int_{0}^{H}\left\langle\sigma_{i j}^{(3)}\left[\varepsilon_{i j}^{(3)}+\varepsilon_{i j}^{r}\left(x, y, z-H+t_{3}, t_{3}, \beta\right)\right]\right\rangle_{x} \mathrm{~d} z \\
& \left\langle w_{1-3}\right\rangle=\frac{1}{2 H} \int_{0}^{H}\left\{\left\langle\sigma_{i j}^{(1)} \varepsilon_{i j}^{r}\left(x, y, z-H+t_{3}, t_{3}, \beta\right)\right\rangle_{x}\right. \\
& \left.\quad+\left\langle\sigma_{i j}^{(3)}\left[\varepsilon_{i j}^{r}(x, y, z, H, \alpha)-\varepsilon_{i j}^{r}\left(x, y, z-t_{1}, H-t_{1}, \alpha\right)\right]\right\rangle_{x}\right\} \mathrm{d} z .
\end{aligned}
$$


Here $\varepsilon_{i j}^{(k)}$ and $\sigma_{i j}^{(k)}(k=1,3)$ are the strains and the stresses equal to $\varepsilon_{i j}^{e}$ and $\sigma_{i j}^{e}$, respectively, in the $k$ th layer and to zero in the substrate and other layers.

Substitution of (4), (5) and (7)-(13) into (17)-(19) yields:

$$
\begin{aligned}
\left\langle w_{1}\right\rangle=w_{0} & {\left[\frac{1}{1+v} \frac{t_{1}}{H}-\frac{\exp (-2 \alpha H)\left[1-\exp \left(\alpha t_{1}\right)\right]^{2}}{\alpha H}\right] } \\
\left\langle w_{3}\right\rangle=w_{0}\left[\frac{1}{1+v} \frac{t_{3}}{H}-\frac{\left[1-\exp \left(-\beta t_{3}\right)\right]^{2}}{\beta H}\right] & \\
\left\langle w_{1-3}\right\rangle=2 w_{0}\left[\frac{\exp (-\beta H)\left[\exp \left(-\beta t_{1}\right)-1\right]\left[\exp \left(-\beta t_{3}\right)-1\right]}{\beta H}\right. & {\left[\frac{\exp (-\alpha H)\left[\exp \left(-\alpha t_{1}\right)-1\right]\left[\exp \left(-\alpha t_{3}\right)-1\right]}{\alpha H}\right]\langle\cos \alpha \cos \beta\rangle_{x} }
\end{aligned}
$$

where

$$
w_{0}=E \varepsilon_{0}^{2}(1+v) /[2(1-v)]
$$

and $\langle\cdots\rangle_{x}$ means the averaging over coordinate $x$. Direct calculations show that $\langle\cos \alpha \cos \beta\rangle_{x}=\frac{1}{2} \delta_{\alpha \beta}$, with $\delta_{\alpha \beta}$ being the Kronecker symbol equal to 1 , for $\alpha=\beta$, and 0 , for $\alpha \neq \beta$. As a corollary, in the case with $\alpha \neq \beta$, from formula (22) we have $\left\langle w_{1-3}\right\rangle=0$, that is, the compositionally modulated layers 1 and 3 do not elastically interact.

The frequency $\alpha$ of spatial modulation of the layer- 1 composition is supposed to minimize the system energy when layer 1 is completely deposited onto the substrate. That is, $\alpha$ minimizes the energy of a one-layer $(\delta)$ film of thickness $t_{1}$, deposited onto the substrate. Following [30], $\alpha=1.256 / t_{1}$.

As can be seen from formula (22), $\left\langle w_{1-3}\right\rangle$ (and consequently, $\langle w\rangle$ ) has a discontinuity at the point $\beta=\alpha$. In these circumstances, in order to find the lowest value of $\langle w\rangle$, it is convenient to separately consider the cases with $\beta \neq \alpha$ and $\beta=\alpha$. In the case with $\beta \neq \alpha$, the point $\beta=$ $\beta_{m}$ of minimum energy $\langle w\rangle$ (calculated under the condition that $\alpha$ is specified) coincides with that of the mean energy density $\left\langle w_{3}\right\rangle$ of the one-layer film of thickness $t_{3}$, whose composition is modulated with a frequency $\beta$. In this case, according to the results of [30], we have $\beta_{m} t_{3}=$ 1.256. For illustration, the dependence of $\langle w\rangle$ on $\beta$, for $\beta \neq \alpha$, is shown in figure 2 . Since the function $\langle w(\beta)\rangle$ is discontinuous at $\beta=\alpha$, the lowest value of $\langle w(\beta)\rangle$ equals $\left\langle w\left(\beta_{m}\right)\right\rangle$, if the energy difference $\langle\Delta w\rangle=\left\langle w\left(\beta_{m}\right)\right\rangle-\langle w(\alpha)\rangle \leqslant 0$, and $\langle w(\alpha)\rangle$, if $\langle\Delta w\rangle>0$. The value of $\langle\Delta w\rangle$ is never positive, as shown in figure 3 where the dependence of $\langle\Delta w\rangle$ on $t_{1} / H$ and $t_{3} / H$ is presented. Therefore, $\langle\Delta w\rangle$ always reaches its lowest value at $\beta=\beta_{m}$. The characteristic energy difference $\langle\Delta w\rangle$ equals 0 , only if $t_{1}=t_{3}$, that is, if $\beta_{m}=\alpha$. Consequently, in the situation where $t_{1} \neq t_{3}$, the frequency $\beta_{m}$ of the spatial modulation of the layer- 3 composition is different from the frequency $\alpha$ that specifies the compositional inhomogeneities in layer 1 . The characteristic frequency $\beta_{m}$ can be effectively regulated by tuning the layer- 3 thickness $t_{3}$, a technologically controlled parameter.

\section{Concluding remarks}

Here we have suggested a first approximation model that describes nano-wires associated with compositional inhomogeneities in multilayered films. In the framework of the model, a multilayered film consists of alternate layers $\delta$ and $\gamma$ and is deposited onto a thick substrate $\gamma$ (figure 1). The chemical composition of the phase $\gamma$ (substrate and even layers) is spatially homogeneous. The compositional nano-wires are formed in the layers $\delta$, where the chemical composition is assumed to be spatially inhomogeneous causing periodic modulation of the 


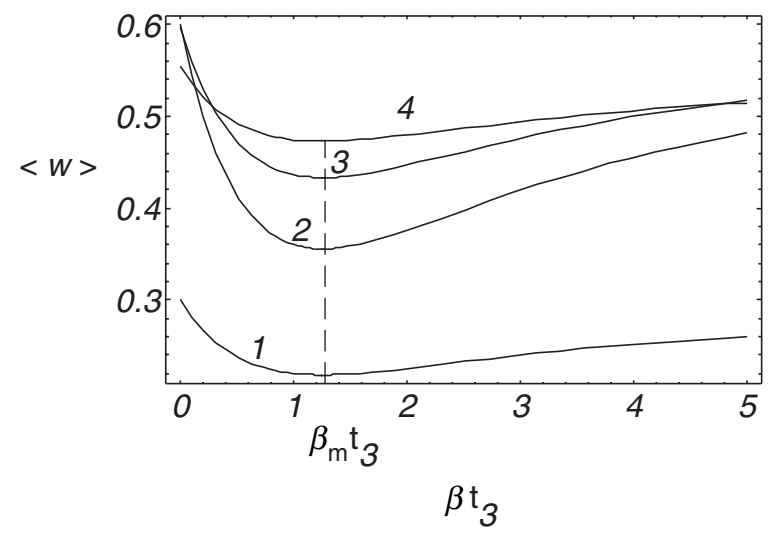

Figure 2. Dependence of $\langle w\rangle$ (in units of $E \varepsilon_{0}^{2}(1+v) /[2(1-v)]$ ) on parameter $\beta t_{3}$, for $\beta \neq \alpha$ and the following values of parameters $t_{1} / H$ and $t_{3} / H: t_{1} / H=t_{3} / H=0.2 ; t_{1} / H=0.2$ and $t_{3} / H=$ $0.6 ; t_{1} / H=t_{3} / H=0.4 ; t_{1} / H=0.6$ and $t_{3} / H=0.2$ (curves $1,2,3$ and 4 , respectively).

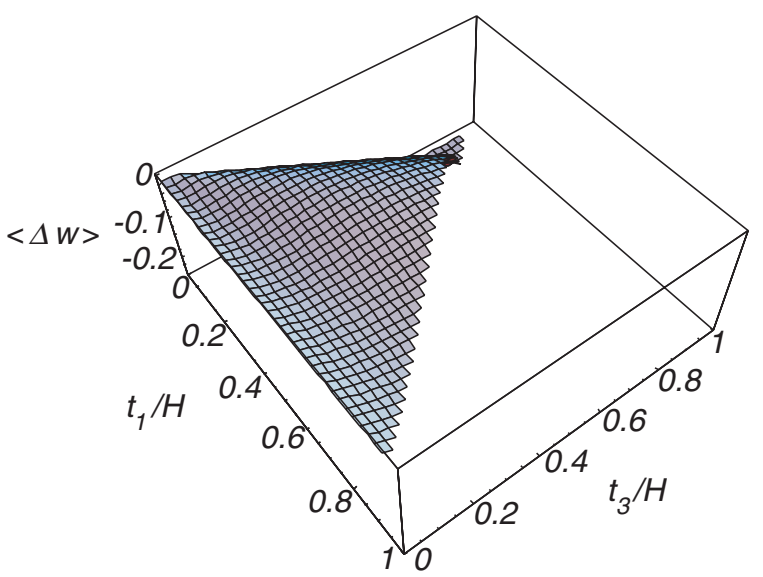

Figure 3. Dependence of $\langle\Delta w\rangle$ (in units of $E \varepsilon_{0}^{2}(1+v) /[2(1-v)]$ ) on $t_{1} / H$ and $t_{3} / H$ in the range of parameters satisfying the condition: $t_{1} / H+t_{3} / H<1$.

crystal lattice parameter. In these circumstances, the characteristic length scales of nanowires associated with compositional inhomogeneities in the layers $\delta$ of the multilayered film strongly depend on the elastic energy density which specifies strains induced by the compositional inhomogeneities and misfit strains generated at the film/substrate interface. In its turn, the elastic energy density in question is dependent on such technologically controlled parameters as layer thickness values and misfit parameters. In this paper we have found general formulae which allow one to calculate the elastic energy density as a function of periods of compositional inhomogeneities in the layers $\delta$ and geometric parameters (layer thickness values) of the multilayered film in the general situation with $(2 N+1)$ layers (see section 3 ). The exact dependence of the elastic energy density on the parameters discussed has been calculated in the exemplary case of three-layer $(N=1)$ films (see section 4$)$.

The results of our theoretical examinations in short are as follows: 
(i) As with the commonly studied situation with one-layer films, the formation of compositional inhomogeneities with desired, from an applications viewpoint, characteristic length scales of the order of a few nanometres is energetically favourable in multilayered films when their geometric parameters are in certain ranges.

(ii) The set of geometric parameters crucially affecting the formation and characteristics of compositional inhomogeneities in multilayered films is richer than that in conventional one-layer films. The set contains $(N+1)$ layer- $\delta$-thickness values.

(iii) In the case of a three-layer film, the spatial frequencies $\alpha$ and $\beta$ that characterize the composition modulation in respectively the first (lower) and third (upper) layers are not identical unless these layers have equal values of the thickness.

These results are important for technological applications of nano-wires associated with compositional inhomogeneities in multilayered films. In particular, point (ii) is worth noting in the context of the technologically interesting possibility of exploiting multilayered films with compositional nano-wires instead of conventional one-layer films. Actually, using a large number of technologically controlled parameters (layer thickness values) of multilayered films, it is possible to provide more flexible and effective control of fabrication and design of compositional nano-wires in multilayered films compared to the conventional situation with one-layer films.

The quantitative results obtained in this paper are approximate. However, they can be used, on the one hand, to estimate the important relationships between the characteristic spatial scales of nano-wires associated with compositional inhomogeneities and geometric parameters of multilayered films and, on the other hand, as a basis for further investigations of nano-wires in multilayered films.

\section{Acknowledgments}

This work was supported (for IAO and AGS) by INTAS (grant no 99-0216), the Russian Foundation of Basic Researches (grant no 01-02-16853) and the Office of US Naval Research (grant no N00014-99-1-0569). The support of the Volkswagen Foundation (research project 05019225) and NATO Collaborative Linkage Grant PST. CLG. 977712 for IAO is also acknowledged.

\section{References}

[1] Roco M C, Williams R S and Alivisatos P (ed) 2000 Nanotechnology Research Directions (Dordrecht: Kluwer)

[2] Edelstain A S and Camarata R C (ed) 1996 Nanomaterials: Synthesis, Properties and Applications (Bristol: Institute of Physics Publishing)

[3] Chow G-M, Ovid'ko I A and Tsakalakos T (ed) 2000 Nanostructured Films and Coatings (NATO Science Series) (Dordrecht: Kluwer)

[4] Siegel R W, Hwu E and Roco M C (ed) 1997 R\&D Status and Trends in Nanoparticles, Nanostructured Materials, and Nanodevices in the United States (Baltimore, MD: International Technology Research Institute)

[5] Chow G-M and Noskova N I (ed) 1998 Nanostructured Materials: Science and Technology (NATO ASI Series) (Dordrecht: Kluwer)

[6] Gleiter H 2000 Acta Mater. 481

[7] Bourett A 1999 Surf. Sci. 43237

[8] Ledentsov N N, Ustinov V M, Shchukin V A, Kop'ev P S, Alferov Zh I and Bimberg D 1998 Semiconductors 32343

[9] Shchukin V A and Bimberg D 1999 Rev. Mod. Phys. 711125

[10] Ustinov V M 2000 Nanostructured Films and Coatings (NATO ARW Series) ed G M Chow, I A Ovid'ko and T Tsakalakos (Dordrecht: Kluwer) pp 41-54

[11] Kim S B, Park S J, Ro J R and Lee E H 1996 J. Cryst. Growth 164356 
[12] Kumada S, Suga S and Ayao O 1996 Phys. Rev. B 5312979

[13] Lee S C, Kang Y B, Kim D C, Ryu J Y, Kang N L and Choi S D 1997 Phys. Rev. B 556719

[14] Mattila T, Wang L-W and Zunger A 1999 Phys. Rev. B 5915270

[15] Kamins T I, Carr E C, Williams R S and Rosner S I 1997 J. Appl. Phys. 81211

[16] Chaparro S A, Drucker J, Zhang Y, Chandrasekhar D, McCartney M R and Smith D J 1999 Phys. Rev. Lett. 83 1199

[17] Chaparro S A, Zhang Y, Drucker J and Smith D J 2000 J. Appl. Phys. 872245

[18] Tersoff J, Teichert C and Lagally M G 1996 Phys. Rev. Lett. 761675

[19] Liang C-T, Pepper M, Simmons M Y, Smith C G and Ritchie D A 2000 Phys. Rev. B 619952

[20] Romanov A E, Petroff P M and Speck J S 1999 Appl. Phys. Lett. 742280

[21] Ovid'ko I A and Sheinerman A G 2001 Appl. Phys. A at press

[22] Strassburg M, Kutzer V, Pohl U W, Hoffmann A, Broser I, Ledentsov N N, Bimberg D, Rosenauer A, Fisher U, Gerthsen D, Krestnikov I L, Maximov M V, Kop'ev P S and Alferov Zh I 1998 Appl. Phys. Lett. 72942

[23] Floro J A, Sinclair M B, Chason E, Freund L B, Twesten R D, Hwang R Q and Lucadamo G A 2000 Phys. Rev. Lett. 84701

[24] Sutter P and Lagally M G 2000 Phys. Rev. Lett. 844637

[25] Liu N, Tersoff J, Baklenov O, Holmes A L and Shin C K 2000 Phys. Rev. Lett. 84334

[26] Liu C P, Gibson J M, Kamins T I, Basile D P and Williams R S 2000 Phys. Rev. Lett. 841958

[27] Jesson D E, Kästner M and Voigtländer B 2000 Phys. Rev. Lett. 84330

[28] Cirlin G E, Polyakov N K, Petrov V N, Egorov V A, Denisov D V, Volovik B V, Ustinov V M, Alferov Zh I, Ledentsov N N, Heitz R, Bimberg D, Zhakharov N D, Werner P and Gösele U 2000 Mater. Phys. Mech. 115

[29] Pehlke E, Moll N, Kley A and Shaefer M 1997 Appl. Phys. A 65525

[30] Glas F 1987 J. Appl. Phys. 623201

[31] van der Merve J H 1991 Crit. Rev. Solid State Mater. Sci. 17187

[32] Fitzgerald E A 1991 Mater. Sci. Rep. 787

[33] Robert A and Kiely C 1991 Phys. Rev. B 441154

[34] Jain S C, Harker A H and Cowley R A 1997 Phil. Mag. A 751461

[35] Ovid'ko I A 2000 Rev. Adv. Mater. Sci. 161

[36] Hirth J P 2000 Acta Mater. 4893

[37] Spaepen F 2000 Acta Mater. 4831

[38] Jain S C, Gosling T J, Willis J R, Totterdell D H J and Bullough R 1992 Phil. Mag. A 651151

[39] Gosling T J, Bullough R, Jain S C and Willis J R 1993 J. Appl. Phys. 738267

[40] Gosling T J, Jain S C, Willis J R, Atkison A and Bullough R 1992 Phil. Mag. A 66119

[41] Gosling T J and Willis J R 1994 Phil. Mag. A 6965

[42] Ovid'ko I A 2000 Nanostructered Films and Coatings (NATO ARW Series) ed G-M Chow, I A Ovid'ko and T Tsakalakos (Dordrecht: Kluwer) pp 231-46

[43] Ovid'ko I A 1999 J. Phys.: Condens. Matter 116521

[44] Gutkin M Yu, Ovid'ko I A and Sheinerman A G 2000 J. Phys.: Condens. Matter 125391

[45] Leónard F and Desai R C 1997 Phys. Rev. B 564955

[46] Leónard F and Desai R C 1998 Phys. Rev. B 588217

[47] Tersoff J 1996 Phys. Rev. Lett. 772016

[48] Ipatova I P, Malyshkin V G, Maradulin A A, Shchukin V A and Wallis R F 1998 Phys. Rev. B 5712968

[49] Rich D H, Tang Y and Liu H T 1997 J. Appl. Phys. 816837

[50] Brunner K B, Zhu J, Abstreiter G, Kienzle O and Ernst F 2000 Thin Solid Films 36939

[51] Cahn J W 1961 Acta Metall. 9795

[52] Ipatova I P, Malyshkin V G and Shchukin V A 1993 J. Appl. Phys. 747198

[53] Wang Y Q, Wang Z L, Brown T, Brown A and May G 2000 Appl. Phys. Lett. 77223

[54] Ovid'ko I A and Sheinerman A G 2001 Phil. Mag. A submitted 